$\xi=-1$

\title{
Korea's Innovative Clusters and Development Strategies Focusing on the 'Loosely Coupled' Cooperation between the Daedeokinnopolis and the Pangyotechnovalley
}

\author{
Won Il Lee* \\ ${ }^{I}$ Department of Business Administration, Hanbat National University, South Korea \\ *Corresponding author E-mail:tech201@ hanbat.ac.kr
}

\begin{abstract}
In this paper, I review current status and development strategies in Korean innovation cluster. In particular, I compared the innovation clusters of Daejeon and Gyeonggi - do, and examined the differences and ways of cooperation. Among Daejeon and Gyeonggi-province innovation clusters, the DaedeokInnopolis is focused on R \& D and pursues technology commercialization in connection with venture companies. Pangyo Techno Valley is a state-of-the-art IT technology centered on high technology companies. If wide-scale cooperation measures are established between Daejeon and Gyeonggi-do clusters, which are different from each other in the process of formation and complexity, a great synergy will be created at the national level. The future development strategy will be derived by examining the representative innovation cluster in Korea. The first strategic direction is to set up a vision of 'loosely coupled' between the Daejeon and Gyeonggi-do innovation clusters. The second strategic direction is to promote 'loosely coupled' wide-area cooperation programs between clusters. The third strategic direction is to foster an open innovation venture ecosystem.
\end{abstract}

Keywords: business, innovation, cluster, valley, future

\section{Introduction}

In today's rapidly changing business environment, technological innovation is an important growth factor for companies. Through such technological innovations, companies can continue to grow. Innovation clusters at the national level are attracting attention, making it possible for them to innovate more rapidly in the region (Klein 2003, Cooke, Uranga, \&Etxebarria 1997, Porter 1998). There is a focus on building innovative clusters that enable these technological innovations in the region. In order to strengthen corporate competitiveness, each country is making efforts to create a technology innovation cluster. Technology innovation clusters are a region where technological innovation is created while various entities gather for mutual interaction(Innovation 1999, Klein 2003, Cooke, Uranga, \&Etxebarria 1997, Porter 1998). The importance of these innovation clusters is expected to become more important in the future.

Generally, clusters are developing around specific industries. For example, industrial clusters can be established for the chemical industry, the shipbuilding industry, and the steel industry respectively. These clusters can be an important infrastructure that drives technological innovation in the industry, in addition to simply gathering relevant innovation organizations in each industry. In other words, the capacity, resources, and technology of the industry are exchanged within these clusters, creating new added value(Innovation 1999, Klein 2003, Cooke, Uranga, \&Etxebarria 1997, Porter 1998).

Governments around the world are aware of the importance of innovation clusters and are working to foster innovation clusters at the national level. Innovation cluster policy is recognized as a very useful science and technology policy. For this reason, many countries are making efforts for regional innovation and industrial innovation through innovation cluster policies. Several countries, including the US, Europe, China, and Japan, are promoting such innovation cluster policies. Silicon Valley is a representative innovation cluster(Innovation 1999, Klein 2003, Cooke, Uranga, \&Etxebarria 1997, Porter 1998).

In Korea, the importance of innovation cluster policy was recognized and various related cluster policies were promoted. Various innovation clusters have been established in different regions of Korea. DaedeokInnopolis in Daejeon, Innovation Cluster in Gyeonggi Province, Innovation Cluster in Daegu, Ulsan and Gwangju are important examples. Through these innovative cluster policies, local innovation is being promoted in the region(Innovation 1999, Klein 2003, Cooke, Uranga, \&Etxebarria 1997, Porter 1998).

This study compares the DaedeokInnopolis with the Pangyo Techno Valley, a major innovation cluster in Gyeonggi Province. DaedeokInnopolis is the largest innovation cluster in Korea and has been promoted for a long time. Pangyo Techno Valley in Gyeonggi-do is a cutting-edge IT-based innovation cluster and a fast-growing innovation cluster in a short period of time. In other words, this paper examines the status and development strategies of Korean innovation clusters. In particular, I will compare the innovation clusters of Daejeon and Gyeonggi - do and examine the differences and cooperation measures.

DaedeokInnopolis, an important innovation cluster in Daejeon, was built with a formation period of more than 40 years. Currently, the government is also building an international scientific business belt based on the DaedeokInnopolis(Won 2012, Lim, Kim \&Yoo 
Jung Hwa, 2004, Kwi, Won 2014, MKE 2011, DaedeokInnopolis 2018). Innovative clusters in Gyeonggi Province include Pangyo Techno Valley, Gwanggyo Techno Valley, and Ansan Science Valley. Pangyo Techno Valley has developed rapidly in advanced IT center over the past decade(Won 2011, 2012, Won \& Lim 2012, pangyotechnovalley 2018).

In DaedeokInnopolis, various research institutes are integrated. DaedeokInnopolis pursues technology commercialization in which research results of research institutes are linked with venture companies. In Pangyo Techno Valley, high technology companies are integrated.

It is important to make a comparison between the DaedeokInnopolis, which has been promoted for a long time, and the rapidly growing Pangyo Techno Valley in a short period of time. In addition, it is very important to consider what has an important impact on the development and formation of innovative clusters. It is desirable to seek mutual cooperation among these heterogeneous innovation clusters. If wide-scale cooperation measures are established between Daejeon and Gyeonggi-do clusters, which are different from each other in the process of formation and complexity, a great synergy will be created at the national level.

\section{Literature Review}

\subsection{Innovation Cluster}

A cluster basically refers to an enriched zone where organizations that create innovation are organically connected with one another (Innovation, B. 1999). The Organization for Economic Cooperation and Development defined a cluster as a network of firms with strong independence, knowledge-producing agencies like universities and research institutes, and customers that are all connected to a production chain that creates added value (Innovation, B. 1999).

The physical and geographic space in which these collaborations can take place is important. It is the cluster concept that has been considered from this spatial point of view.

It is a cluster where the various organizations are collectively clustered(Innovation 1999, Klein 2003, Cooke, Uranga, \&Etxebarria 1997, Porter 1998). In various organizations conducting global innovation research, these clusters have been studied intensively from the past.

Many innovation clusters, including Silicon Valley in the United States, medical clusters in the Boston area, and research triangle park in North Carolina, have been noted for leading venture startup ventures and innovation. Innovative clusters such as China's Zhongguancun and Singapore's One North Biopolis have received considerable attention.

The main purpose of these clusters is for innovation. At present, technological innovation has become an important issue and technological innovation has been the most important factor in environmental change. These innovation clusters are the areas where universities, research institutes, venture companies and related support service agencies are integrated to create innovation(Innovation 1999, Klein 2003, Cooke, Uranga, \&Etxebarria 1997, Porter 1998). In the future, the innovation cluster will become even more important based on the creation of national competitive advantage. Innovation clusters are not only for creating competitive advantage in geographical areas, but also a growth platform for technological innovation of companies. Through this integrated network of technological innovations, enterprises can constantly create competitive advantage and grow to the stage of growth. Entrepreneurs can create new innovations through entrepreneurship in innovation clusters. In addition, research institutes, universities and such entrepreneurs can cooperate in innovation clusters and create new innovations(Innovation 1999, Klein 2003, Cooke, Uranga, \&Etxebarria 1997, Porter 1998).

These innovative clusters need to be considered separately from industrial clusters. Industrial clusters can be said to build clusters by industry sector. It can be said that industrial clusters create synergy by integrating front and back industries by industry sector Prior to the research of innovation clusters, much research has been done on clusters built by these industries. It is a technology commercialization ecosystem that creates synergy by integrating companies, research institutes, universities, and venture investments within these industrial clusters.

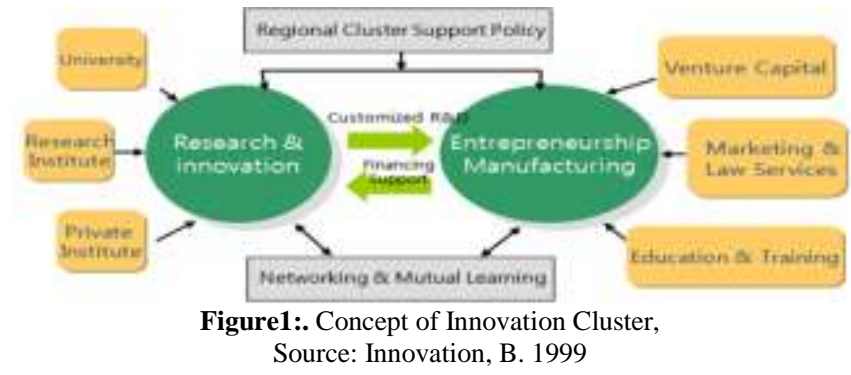

\subsection{Innovation Cluster and Innovative Ecosystem}

Innovation clusters that focus on technological innovation are getting more attention in the 21 st century management environment where technological innovation is important(D'Aveni 1994, Chung 2011, Betz 2011, Schiling 2010). These innovation clusters play a role in a single ecosystem beyond what has been built. Innovation clusters are at the center when the region is the unit of competition.

These innovative clusters are ecosystems that form a virtuous cycle of corporate creation and fostering and destruction in the region. Ecosystems have positive and negative aspects. On the positive side, the various entities interact and form a virtuous circle. On the negative side, ecosystems are subject to extreme competition. The positive and negative aspects of these ecosystems can be said to occur simultaneously in innovation clusters. Innovation clusters as such are very dynamic. Under such a dynamic environment, new companies are created, grown and competed with each other.

Activation policies are required to maintain the dynamism of these clusters. Innovation clusters are a cluster of universities, research institutes, venture companies, and related support organizations that create technological innovation through mutual networks(Innovation 1999, Klein 2003, Cooke, Uranga, \&Etxebarria 1997, Porter 1998). Much research has been conducted on these innovation clusters. In particular, OECD has published many reports emphasizing the importance of innovation clusters as an important part of innovation policy. Professor Michael Porter of Harvard University also stressed the importance of innovation clusters and emphasized the importance of a regional cooperative network to create innovation(Innovation 1999, Klein 2003, Cooke, Uranga, \&Etxebarria 1997, Porter 1998).

\subsection{DaedeokInnopolis and Gyeonggi-Province Innova- tive Cluster Literature Study}

Lee W.I.(2012) has studied the direction and strategy of DaedeokInnopolis as a technology innovation cluster. In particular, it concentrated on the aspect of successful diffusion of the R \& D special zone system in Korea as well as Daedeok, which has the headquarter of DaedeokInnopolis.

Lim, D.S.(2004) discusses the development direction of the DaedeokInnopolis as a technological innovation cluster and suggests future direction of the technology innovation cluster. 
Kwon Ki-hwan, Lee W.I. (2014) discussed the concept of creative economy and the current status and improvement direction of DaedeokInnopolis. In order to continuously develop DaedeokInnopolis, the largest innovation cluster in Korea, DaedeokInnopolis needs to build a creative economy infrastructure. First, DaedeokInnopolis is created as a creative economic ecosystem. The second is to further strengthen the support programs that will gradually foster the company. Thirdly, it is necessary to strengthen the financial support for these companies and to create an environment to secure investment opportunities.

Lee W.I., Lim, D.S., Lee, Y. H., \&Jeong E. J.(2011) mentioned the importance of Pangyo Techno Valley as a technological innovation cluster and suggested ways to foster technology innovation clusters through scenario planning methodology.

Lee W.I.(2012) noted the importance of regional science and technology policies and pointed out the importance of innovation clusters. It is the innovation cluster that is the center of the regional innovation system and it is mentioned that the innovation cluster policy is very important in the region. In this paper, Lee(2012) analyzed the technological innovation system in the metropolitan area and the process of change of science and technology policy in Gyeonggi - do. Gyeonggi-do innovation clusters include Gwanggyo Techno Valley, Pangyo Techno Valley, and Ansan Science Valley, and point out that these innovation clusters should be developed through organic cooperation. In order to develop innovative clusters in Gyeonggi Province, it is necessary to strengthen capacity for science and technology management at the first regional level, and to foster a global innovation cluster considering the second regional ecosystem. Third, It is pointed out that strengthening industry-academia cooperation is needed.

Lee W.I., Lim, D.S.(2012) mentioned that Pangyo Techno Valley, Gwanggyo Techno Valley, and Ansan Science Valley should be developed not only in terms of hardware but also in terms of software as a strategy for developing innovative clusters in Gyeonggi Province. It is mentioned that not only hardware upbringing such as building construction, cluster complex construction, but also overseas marketing, overseas technology commercialization strategy should be established. Based on these literature studies and practical field case studies, the following analysis is conducted.

\section{Analysis of Korea's Innovation Cluster}

\subsection{DaedeokInnopolis in Daejeon}

In Korea, since the Daedeok R \& D institute started in 1973, interest in innovative clusters has increased and construction has begun(Won 2012, Lim, Kim \&Yoo Jung Hwa, 2004, Kwi, Won 2014, MKE 2011, DaedeokInnopolis 2018). The DaedeokInnopolis started in 1973 with government-funded research institutes and several companies. In 2004, special laws for the creation of the DaedeokInnopolis were established and special research and development zones were designated. In the second stage of DaedeokInnopolis development, research institutes, venture companies, and universities can be considered as clusters for innovation that can develop in synergy(Won 2012, Lim, Kim \&Yoo Jung Hwa, 2004, Kwi, Won 2014, MKE 2011, DaedeokInnopolis 2018). In DaedeokInnopolis, innovative clusters have been established centered on government-funded research institutes and efforts have been made to commercialize technical achievements to create economic added value The R \& D Special Zone has completed Phase 1 for the establishment of innovative clusters from 2004 to 2012 and entered Phase 2 of the DaedeokInnopolis from 2012. This two-stage model can be said to extend this success routine to other regions based on this success model(Won 2012, Lim, Kim \&Yoo Jung Hwa, 2004, Kwi, Won 2014, MKE 2011, DaedeokInnopolis 2018). In addition to the DaedeokInnopolis, it will support the expansion of models such as Busan, Gwangju and Daegu to foster clusters centered on technological innovation in other regions. Innovation clusters in other regions can be said to promote local customized technology development strategies to reflect local needs. DaedeokInnopolis has more than 1,200 venture companies. In these special zones, various technology commercialization programs are operated for these companies. Daejeon is currently constructing an international science business belt, which is building an innovative cluster centered on basic science focusing on basic science competence. It is the concept of building an innovation cluster centered on basic science covering DaedeokInnopolis(Won 2012, Lim, Kim \&Yoo Jung Hwa, 2004, Kwi, Won 2014, MKE 2011, DaedeokInnopolis 2018).

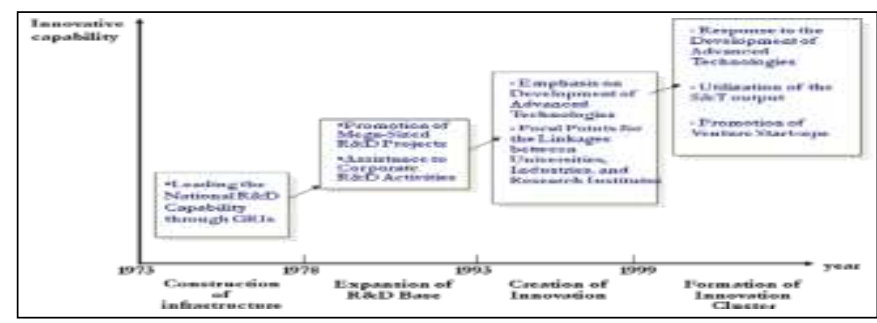

Figure2:. Changing Roles of DaedeokInnopolis, (Source: DaedeokInnopolis)

\subsection{Pangyo Techno Valley in Gyeonggi Province}

In the metropolitan area, which has a population of 25 million people, the importance of innovation clusters is becoming more and more important. In the past, the traditional industrial area, called Guro Industrial Complex, was transformed into an innovation cluster. In the past, garbage dumps were transformed into media innovation cluster Sangam DMC. In Gyeonggi Province, an innovation cluster was established in conjunction with the development of a new city. These innovation clusters include Pangyo Techno Valley, Gwanggyo Techno Valley, and Ansan Science Valley. The Gwanggyo Techno Valley was constructed to construct the Gwanggyo Techno Valley in order to create a space where new towns such as high-tech and apartment buildings can be united instead of bed town. Gwanggyo Techno Valley has several high-tech convergence research institutes, bio-research institutes, and nano research institutes. It has potential to grow as a base for advancing biotechnology in connection with hospitals nearby.

Pangyo Techno Valley enjoys a very close local superiority, $12 \mathrm{~km}$ away from Seoul. It is being transformed into a point of important technology that manpower is gathering. Pangyo Techno Valley is growing as a forward base for the global IT convergence industry based on the accumulation effect of IT companies(Won 2011, 2012, Won \& Lim 2012, pangyotechnovalley 2018). Pangyo Techno Valley is a cluster that fosters venture businesses based on advanced IT technology. In Pangyo Techno Valley, innovative clusters have been successful in a very short time and have received worldwide attention. With the development of advanced IT technology such as artificial intelligence and Internet of the future, the development of Pangyo Techno Valley is more promising.

Pangyo Techno Valley has been established since 2005 as a technology innovation cluster in Pangyo New Town. With Gwanggyo, Ansan, and Gwacheon as the main technological innovation infrastructure of Gyeonggi Province, it has the goal of nurturing it as a global R \& D hub centered on IT convergence technologies. There are public support centers, global R \& D centers, and industryacademia-research $\mathrm{R} \& \mathrm{D}$ centers for the support of tenant companies in Pangyo Techno Valley. Pangyo Techno Valley's major tenants are Korea Pasteur Research Institute, SK Chemicals, SK Telesys, LIG Nexwin, AhnLab Consortium and Webzen(Won 2011, 2012, Won \& Lim 2012, pangyotechnovalley 2018) 
Table 1:. Framework for Analysis: Comparison between DaedeokInnopolis and Pangyo Techno Valley (Source: innopolis,pangyotechnovalley)

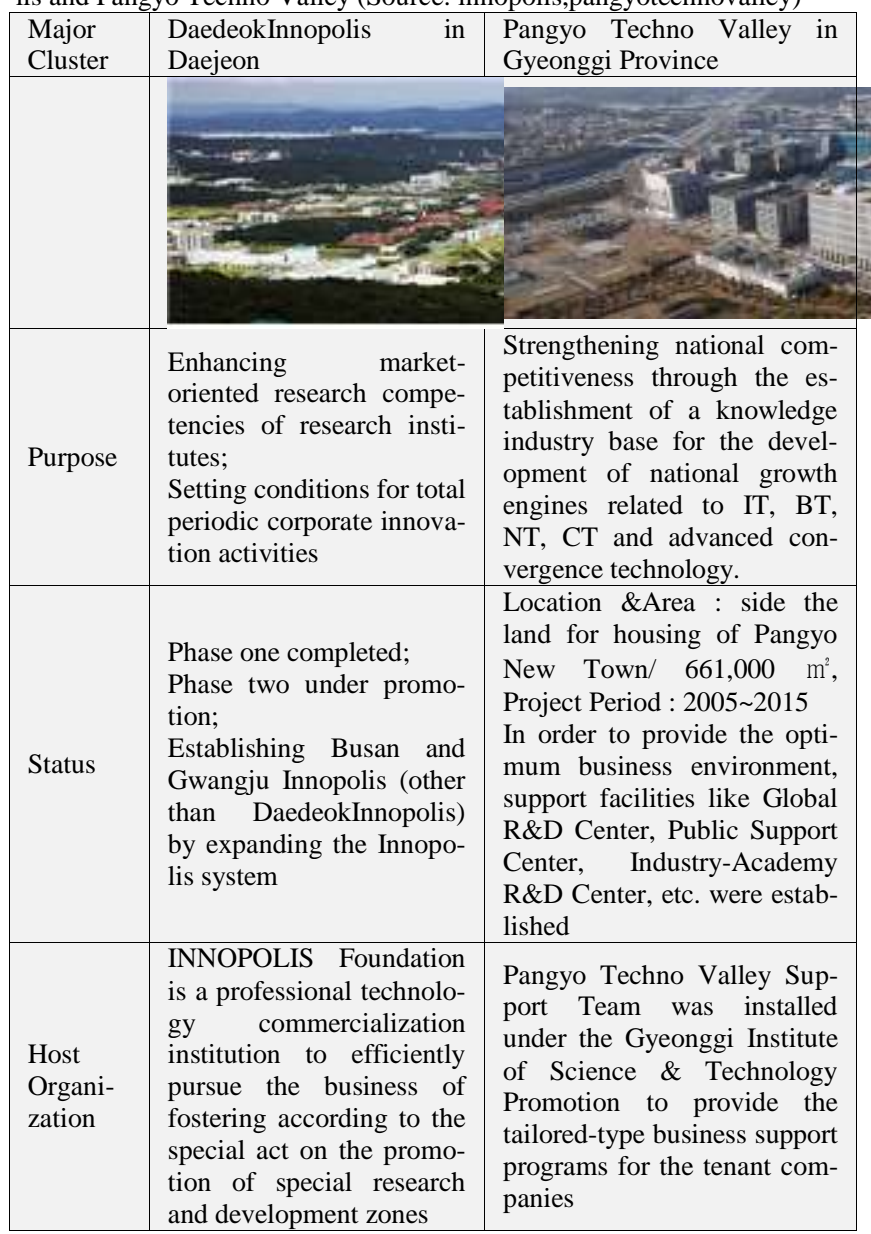

\section{Strategic Direction for Korea's Innovation Cluster Development}

In this paper, I use the scenario planning methodology to establish the strategic direction for the development of Korean innovation cluster, and draw up the future direction and strategic direction of Korea 's innovation cluster. To set strategic directions to build a creative innovation cluster, the future conditions at the regional level can be forecasted using scenario planning methodology.

The foresight method is used to forecast the future at the regional level. Foresight is strategic plan setting related to the networking of experts through future studies of various literature and papers as well as multiple future tools. In this study, strategic directions for co - development between DaedeokInnopolis and Pankyo Techno Valley are derived by utilizing the scenario planning methodology which is widely used for foresight method. The development direction of the cluster is largely divided into cluster management capability analysis axis and 'loosely coupled' cooperation' axis. Through this analysis, I can derive the future of Korea 's innovation cluster. To consider uncertainty, relevant issues were examined through workshops, forums, public hearings, papers, and interviews with experts related to the DaedeokInnopolis and Pangyo Techno Valley.

Considering these uncertainties, this study categorizes them into an uncertainty axis related to building the internal competencies of each cluster and 'loosely coupled' cooperation'. Based on these uncertainty issues, four credible scenarios for 2030 were established in Figure 1. The 2 horizontal axis is internal capability of each cluster and the vertical axis is connection, cooperation, and coordination between cluster.

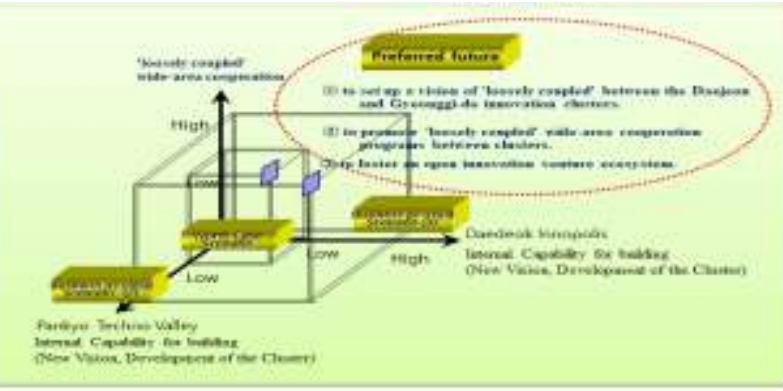

Figure 3:. 3 Dimensional Scenario Planning for Preferred Future (Desirable Future)

The Korean Innovation Cluster derives three major strategic directions to achieve this desirable future scenario.

The first strategic direction is to set up a vision of 'loosely coupled' between the Daejeon and Gyeonggi-do innovation clusters. The vision for cooperation between Daejeon and Gyeonggi-do innovation clusters from a long-term perspective is set up and step-by-step fostering plans are set up. It is the long-term vision set-up in the innovation cluster, the step-by-step development according to the vision setting, and the wide-ranging cooperation plan between the innovation cluster.

The second strategic direction is to promote 'loosely coupled' wide-area cooperation programs between clusters. Pangyo Techno Valley is focused on high technology business, and DaedeokInnopolis and International Science Business Belt can be regarded as science-oriented integrated complex. These heterogeneous complexes are located in Daejeon and Gyeonggi-do. Therefore, it is necessary to provide a wide range of cooperation programs so that they can cooperate with each other not only in one area but also in the belt type between Gyeonggi and Daejeon. For this purpose, it is necessary to establish a cooperative program system, to grasp the needs for cooperation, and to use cooperation programs among the clusters. For example, companies in Daejeon can enter the Pangyo Techno Valley using the cooperation program and then enter the global market. In addition, high-tech enterprises in Pangyo Techno Valley can cooperate with research institutes of DaedeokInnopolis by using cooperation programs, which can further strengthen their technological capabilities.

The third strategic direction is to foster an open innovation venture ecosystem. DaedeokInnopolis was established at the beginning of the government research institute, and venture companies were integrated to commercialize these achievements. Pangyo Techno Valley and Gwanggyo Techno Valley formed a venture complex centered on IT technology and biotechnology in Gyeonggi Province. Since it is the enterprise that creates the actual economic value, it is necessary to prepare various venture business incubation programs so that the innovation cluster can be operated not by the research institute but by the venture business center. If an innovation cluster is operated centering on the research center, it can be transformed into a research institute city for research only. Therefore, a step-by-step nurturing program is prepared and supported so that the venture company can grow as a global company after the start-up stage. As venture companies grow through open innovation, various support programs centering on venture companies should be established and operated in order to cooperate with universities near research institutes.

\section{Summary and Conclusions}

In this paper, I have reviewed current status and cooperative development strategies in Korea 's innovation cluster. In particular, I compared the innovation clusters of Daejeon and Gyeonggi province and examined ways of mutual cooperation. Daejeon Innova- 
tive Cluster, DaedeokInnopolis, was built over 40 years. Among the Innovation Clusters in Gyeonggi Province, Pangyo Techno Valley developed rapidly in advanced IT center during the past 10 years. DaedeokInnopolis starts with the center of research institute and pursues technology commercialization in cooperation with venture companies. If wide-scale cooperation measures are established between Daejeon and Gyeonggi-do clusters, which are different from each other in the process of formation and complexity, a great synergy will be created at the national level. Strategies for cooperative development of innovative clusters in Daejeon and Gyeonggi Province are as follows.

The first strategic direction is to set up a vision of 'loosely coupled' between the Daejeon and Gyeonggi-do innovation clusters. The vision for cooperation between Daejeon and Gyeonggi-do innovation clusters from a long-term perspective is set up and step-by-step fostering plans are set up.

The second strategic direction is to promote 'loosely coupled' wide-area cooperation programs between clusters. It is necessary to provide a wide range of cooperation programs so that they can cooperate with each other not only in one area but also in the belt type between Gyeonggi and Daejeon.

The third strategic direction is to foster an open innovation venture ecosystem. As venture companies grow through open innovation, various support programs centering on venture companies should be established and operated in order to cooperate with universities near research institutes

\section{References}

[1] Chung 2011, Strategic Technology Management, ParkYongSa, 3nd edition, 2011, pp 397-443.

[2] Cooke, P., Uranga, M. G., \&Etxebarria, G. 1997, Regional innovation systems: Institutional and organisational dimensions. Research policy, 26(4-5), 475-491.

[3] Frederick Betz 2011, Managing Technological Innovation, Competitive Advantage from Change, John Wiley \& Sons

[4] Innovation, B. 1999, The Cluster Approach. OECD Proceedings. OECD Publication Service, 418.

[5] Kwi Hwan Kwon, Won I. Lee 2014, A Study on the DaedeokInnopolis as a Creative Economy Infrastructure, Innovation Cluster Research, Vol. 6, No. 1.

[6] Lim Deok Soon, Kim Wang Dong, Yoo Jung Hwa. 2004, Development process and international comparison of Daedeok Science Town: - From the perspective of innovation cluster. Journal of Technology Innovation, Vol. 7, No. 2.

[7] Melissa A. Schiling 2010, Strategic Management of Technological Innovation, Third Edition, McGrawHill.

[8] Richard A. D'Aveni 1994, Hyper-Competition, Simon \& Schuster.

[9] Won I. Lee 2012, A Study on the Strategy for the R \& D Special Zone as a Global Innovation Cluster. Journal of the Korean Academic Society of Industrial Science, Vol. 13 No. 11, pp 5015-5024.

[10] Won I. Lee, Lim Deok Soon, Lee, YounHee, \&JeongEuiJeong 2011, A Study on the Strategic Direction of Technology Innovation Cluster Construction, Journal of Technology Innovation, Vol 14, No. 2, pp 301-319.

[11] Won I. Lee 2012, Research on Science and Technology Innovation Policies at the Local Level - Focusing on Science and Technology Policy and Industry-University Cooperation in Gyeonggi Province, Innovation Cluster Research Vol. 4, No. 1.

[12] Won I. Lee, Lim Deok Soon 2012, Gyeonggi-do innovation cluster development strategy Lee, Innovation cluster research Vol. 4, No. 1

[13] Klein, J. 2003, Innovative clusters: Drivers of national innovation systems.

[14] Porter, M. E. 1998, Clusters and the new economics of competition (Vol. 76, No. 6, pp. 77-90). Boston: Harvard Business Review.

[15] MKE, 2011, 2 phase Master Plan for the development of Development of the Innopolis.

[16] DaedeokInnopolis (n.d.) Retrieved March 1 2018, from http://www.innopolis.or.kr

[17] pangyotechnovalley (n.d.) Retrieved March 1 2018, from http://www.pangyotechnovalley.org 